\title{
Bone metastatsis from differentiated thyroid cancer treated successfully with I131. Case report
}

Authors: ${ }^{1}$ Entela ,Puca, ${ }^{2}$ Ema Lumi, ${ }^{3}$ Blertina Olldashi, ${ }^{4}$ Sonila Bitri, ${ }^{5}$ Kadir Burak Koza, ${ }^{6}$ Arben Dhima, ${ }^{7}$ Edmond Puca, 8 Agron Ylli.

${ }^{1}$ Endocrinologist, American Hospital, Tirana, AlbaniaHospital

'Endocrinologist,Department of internal Medicine, Regional Hospital "Teni Konomi",Korce,Albania..

${ }^{3}$ Endocrinologist, Neo Style Clinic, Tirana Albania.

${ }^{4}$ Toxicologist, American Hospital,Tirana Albania.

${ }^{5}$ General Surgery. American Hospital,Tirana Albania

${ }^{6}$ Radiologist. American Hospital,Tirana Albania

'Infectologue, UHC “ Mother Teresa”, Tirana Albania

8 Endocrinologist, Head of Endocrine Department, UHC "Mother Teresa",Tirana,Albania

\section{Introduction}

Thyroid cancer is a very common malignancy and fastest increasing of all cancer all over the world Differentiated thyroid cancer (DTC) in general has a good prognosis when ist diagnosed early. Late stage disease with Bone metastasis may cause severe complications, increases mortality rate, decreases the quality of life, and shortens the patients' survival. The treatment is still controversial. Surgery or radioiodine therapy alone is usually unsatisfactory, thus the management needs multidisciplinary collaboration.

\section{Case Presentation}

We report a case of a 40 year-old female presented to American Hospital, in August 2012, presenting with a complaint of the progressive swelling of her neck for the last year on left side. Her past history was right thyroid lobectomy for multiple nodules at the age of 28 , biopsy was microfollicular adenoma. she had palpable mass in the right side of the neck that corresponded tohypoechoic nodule $1,58 \times 1,73 \times 2,15 \mathrm{~cm}$ seen in the thyroid ultrasound with increased flow on color Doppler. Her serum thyroid-stimulating hormone (TSH) was 1,45 and thyroglobulin $(\mathrm{Tg})$ was $948,9 \mu \mathrm{g} / \mathrm{L}$ (reference range: $1-100 \mu \mathrm{g} / \mathrm{L}$ ). kalcitonin was normal. Thyroid scan showed a cold nodule left lobe. FNA-cytology was negative for malignancy. The patient was elected to proceed with completion thyroidectomy. Surgical pathology showed Multifocal papillary thyroid cancer 1,4 cm with no vascular or capsular invasion. On September 2012 four weeks after surgery she received $100 \mathrm{mCi}$ and underwent post treatment whole body scan revealed uptake on thyroid bed and intense activity in the region of the pelvis. Pelvic CT showed litic lesion in sacral bone. She started treatment with Levothyroxine 175 mcg/day. On April 2013 four weeks without treatment TSH was132,4 Thyroglobulin 0,1 and the thyroglobulin antibodies (anti-TG) were negative. Diagnostic whole body scan with $5 \mathrm{mCi}$ showed resolution of the previously seen areas of uptake within the thyroid bed and pelvic area. On October 2015 she is on treatment with LT4 $175 \mathrm{mcg} / \mathrm{day} \mathrm{Tg} \mathrm{0,01,} \mathrm{TSH}$ 0,05 and negative Anti TG. Pelvic CT showed decreased litic lesion with peripheral sclerotic pattern.

\section{Conclusions:}

This case demonstrates the benefits of RAI therapy even in patients with metastatic thyroid cancer treated successfully only with $I 131$. 